\title{
Coronavirus Disintegration using Ultraviolet Light Therapy
}

\author{
Pradeep Kumar Pasricha \\ Scientist-F (Retired) \\ National Physical Laboratory \\ New Delhi, India \\ pradeep1951@rediffmail.com
}

\begin{abstract}
An ideal model of a cell full of coronaviruses is considered. The propagation of ultraviolet electromagnetic waves in such a dielectric medium is examined to ascertain whether the ultraviolet radiation may deliver energy to the medium. With the known observations of refractive indices of cell and virus, above the ultraviolet wavelengths, it is computed that the ultraviolet radiation in the UVB - UVA band of wavelengths $(280-400 \mathrm{~nm})$ deliver "excessive" energy to the dielectric medium. Thus, light at ultraviolet wavelengths may penetrate tissue and disintegrate coronavirus in cells. Ultraviolet light therapy at narrow range of wavelengths in the UVB - UVA band, for "lower" levels and "briefer" periods of radiation exposure, may reduce severity of conditions in the gravely affected coronavirus patients. The material and equipment may be sanitized with the exposure to ultraviolet radiation in the UVC band of wavelengths (100 - $180 \mathrm{~nm}$ ). The use of "UV protected" glasses is "a must" to safeguard one's eyes from the ultraviolet radiation exposure.
\end{abstract}

\section{Introduction}

The model calculations presented in this report are in view of the website of World Health Organization (WHO), "Ultraviolet radiation and health-WHO" [1]. The electromagnetic spectrum of ultraviolet (UV) radiation in the wavelength band $100-400 \mathrm{~nm}$ is divided into three regions, UVA, UVB and UVC. The corresponding wavelength bands are $315-400 \mathrm{~nm}, 280-$ $315 \mathrm{~nm}$ and $100-280 \mathrm{~nm}$, respectively. The shorter UVC wavelengths penetrate the human skin the least. The narrow band of UVB wavelengths just penetrates the human skin. It is the UVB that has greater impact on human health, both useful and hazardous. The therapeutic use of UVB radiation to treat a number of diseases is well-established [2]. The corresponding therapy is called ultraviolet light therapy. The longer UVA wavelengths penetrate the human skin and tissue. These wavelengths are absorbed, delivering energy to cells.

In the present study, an ideal model of a cell-plus-viruses is envisaged, where a large number of viruses are ideally placed in a cell so as to form an ideal spherical microcavity. The dielectric medium of such an ideal microcavity is examined to determine whether such a dielectric medium may have "excessive" (photon) energy transfer, compared to "normal" energy 
delivery, by the ultraviolet radiation. The corresponding narrow range of wavelengths in the UVB and UVA bands is thus determined.

The present study is a sequel to an earlier study by the author, "A Study toward Disintegration of Coronavirus using Near-infrared Light Therapy" [3]. The near-infrared wavelengths heat tissue for the consequent health-related benefits. The corresponding therapy is called near-infrared light therapy. The relative importance of UV light therapy vis-à-vis nearinfrared light therapy may be determined in terms of "Q" of the microcavity, which has not been calculated. It is worthwhile to note that the (photon) quantum of energy (hv, J) at the ultraviolet wavelength $300 \mathrm{~nm}$ is three times higher than the (photon) quantum of energy (hv, J) at the nearinfrared wavelength $1000 \mathrm{~nm}$, where $\mathrm{h}$ is the Planck's constant, $v$ the electromagnetic wave frequency and $\mathbf{J}$ the energy in joules. One may visualize an analogy between the near-infrared light therapy and the ultraviolet light therapy in terms of the medical process termed cauterization. The cauterization is the process to stop haemorrhage, say, a minor flow of blood from a ruptured blood vessel on skin. It is also used to cauterize infected tissue around a minor skin wound. One may do cauterization by applying a heated instrument, the equivalent of nearinfrared light therapy. One may also do cauterization by fixing the skin wound with silver nitrate crystals and bandaging the wound, the equivalent of ultraviolet light therapy. In the latter process of cauterization the moistening of the silver nitrate crystals leads to a chemical reaction that burns/heats to coagulate tissue on the skin.

\section{Observations}

\section{$\underline{\text { Basic Data on Virus }}$}

Size of virus: $20-400 \mathrm{~nm}$ (nanometer, 1 nanometer $=10^{-9}$ meters) $[4]$

Diameter of virus, assumed spherical, in UVB - UVA band $(280-400 \mathrm{~nm})$ in the present model:

(i) $100 \mathrm{~nm}$

(ii) $200 \mathrm{~nm}$

Radius of virus in the present model:

(i) $50 \mathrm{~nm}$

(ii) $100 \mathrm{~nm}$

Refractive index of virus at $532 \mathrm{~nm}: 1.45$ [5]

Refractive index of virus in UVB - UVA band $(280-400 \mathrm{~nm})$ in the present model: 1.45

Basic Data on Human Cell

Size of cell (nucleus + cytoplasm): $8000 \mathrm{~nm}$

Thickness of cell membrane: $10 \mathrm{~nm}$ 
Thickness of membrane, assumed spherical, in the present model: $10 \mathrm{~nm}$

Thickness of cell, assumed spherical, in the present model: $8000 \mathrm{~nm}$

Refractive index of cell: 1.36 - 1.38 [6]

Refractive index of cell at $510-750 \mathrm{~nm}: 1.33-1.38$ [7]

Refractive index of cell in UVB - UVA band $(280-400 \mathrm{~nm})$ in the present model:

(i) 1.37

(ii) 1.39

\section{Methodology}

(i) Virus radius $r=50 \mathrm{~nm}$, cell refractive index $n=1.37$

The design of an ideal spherical microcavity at $650 \mathrm{~nm}$ of viruses of radius $r=50 \mathrm{~nm}$ (diameter $100 \mathrm{~nm}$ ) and refractive index $n=1.45$ in a cell of refractive index $n=1.37$ is presented in Figure 1.

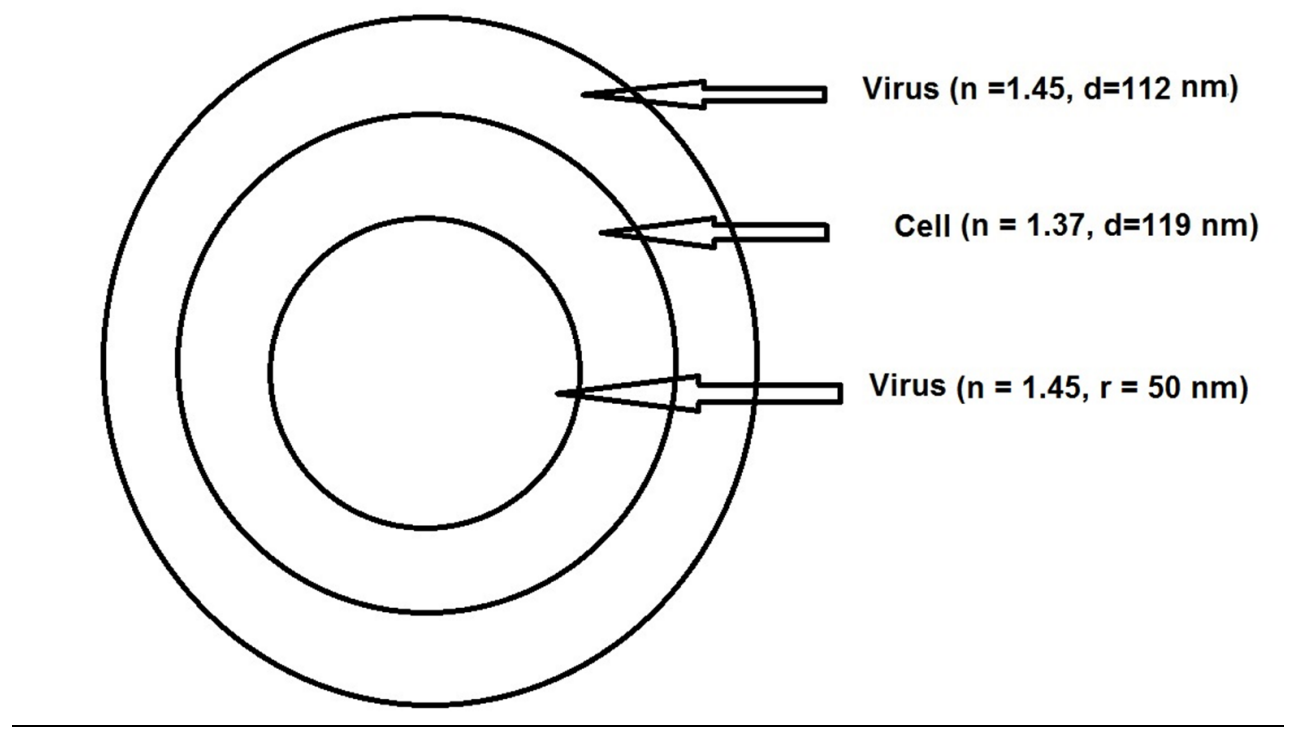

Figure 1. A spherical microcavity consisting of virus-core of radius $r=50 \mathrm{~nm}(\mathrm{n}=1.45)$, and eight pairs of layers of cell $(n=1.37)$ and virus $(n=1.45)$. Two pairs of layers of virus and cell are depicted. There are 17 viruses in a cell, overall.

Such a microcavity is termed as Bragg Onion Resonator without a stem [8]. (A stem is an opening, which is used to excite TE/TM modes of monochromatic electromagnetic waves.) It is also termed as Bragg Reflector [9]. The formulation of a Bragg Onion Resonator for $\mathrm{TE}_{10}$ (and $\mathrm{TM}_{10}$ mode) of electromagnetic wave propagation has been coded in the $\mathrm{C}$ programming language 
[10]. This C programming code has been used to compute the wavelength of resonance in the spherical microcavity in Figure 1. (A similar set of computations can be made for $\mathrm{TM}_{10}$ mode.)

The thickness of virus-layer is $\mathrm{d}=650 / 4 * 1.45 \approx 112 \mathrm{~nm}$, which approximately equals the size of virus-core (diameter) of $100 \mathrm{~nm}$. The thickness of cell-layer is $\mathrm{d}=650 / 4 * 1.37 \approx 119 \mathrm{~nm}$. There are 17 viruses in a cell. The total thickness of the spherical microcavity, including the thickness of cell-membrane, is roughly $4000 \mathrm{~nm}$; the cell size (diameter) is $8000 \mathrm{~nm}$.

The spherical microcavity in Figure 1 implies resonance around wavelength $315 \mathrm{~nm}$. The quality of occurrence of resonance in the microcavity is "high". A resonance in a cavity implies "excessive" transfer of (photon) energy at the computed wavelength. (In the present study, it suffices to qualify the meeting of the condition of occurrence of resonance in a microcavity as "high" or "good" or "fair".) In reality, a band of wavelengths between $312 \mathrm{~nm}$ to $329 \mathrm{~nm}$ reveal resonance from "good"-to-"high"-to-"good". These wavelengths of resonance are almost in the UVA band, by definition.

It may be noted that one cannot design an ideal spherical microcavity at $650 \mathrm{~nm}$ for the virus-core of radius $r=100 \mathrm{~nm}$ (diameter $200 \mathrm{~nm}$ ), since the virus-core size $(200 \mathrm{~nm})$ is not roughly equal to the designed virus-layer thickness $\mathrm{d} \approx 112 \mathrm{~nm}$ at $650 \mathrm{~nm}$.

(ii) Virus radius $r=50 \mathrm{~nm}$, cell refractive index $n=1.39$

The design of an ideal spherical microcavity at $650 \mathrm{~nm}$ of viruses of radius $\mathrm{r}=50 \mathrm{~nm}$ (diameter $100 \mathrm{~nm}$ ) and refractive index $\mathrm{n}=1.45$ in a cell of refractive index $\mathrm{n}=1.39$ is considered.

The thickness of virus-layer is $d=650 / 4 * 1.45 \approx 112 \mathrm{~nm}$, which approximately equals the size of virus-core (diameter) of $100 \mathrm{~nm}$. The thickness of cell-layer is $\mathrm{d}=650 / 4 * 1.39 \approx 117 \mathrm{~nm}$. There are 17 viruses in a cell. The total thickness of the spherical microcavity, including the thickness of cell-membrane, is roughly $4000 \mathrm{~nm}$; the cell size (diameter) is $8000 \mathrm{~nm}$.

The spherical microcavity implies "good" resonance around wavelength $238 \mathrm{~nm}$. A resonance in a cavity implies "excessive" transfer of (photon) energy at the computed wavelength. (In the present study, it suffices to qualify the meeting of the condition of the occurrence of resonance in a microcavity as "high" or "good" or "fair".) In reality, a band of wavelengths between $236 \mathrm{~nm}$ to $240 \mathrm{~nm}$ reveal "good" resonance. These wavelengths of resonance are toward the rear of UVC band, or front of UVB band, by definition.

(iii) Virus radius $r=100 \mathrm{~nm}$, cell refractive index $n=1.37$

The design of an ideal spherical microcavity at $1050 \mathrm{~nm}$ of viruses of radius $r=100 \mathrm{~nm}$ (diameter $200 \mathrm{~nm}$ ) and refractive index $\mathrm{n}=1.45$ in a cell of refractive index $\mathrm{n}=1.37$ is considered.

The thickness of virus-layer is $\mathrm{d}=1050 / 4 * 1.45 \approx 181 \mathrm{~nm}$, which approximately equals the size of virus-core (diameter) of $200 \mathrm{~nm}$. The thickness of cell-layer is $d=1050 / 4 * 1.37 \approx 192$ $\mathrm{nm}$. There are 10 viruses in a cell. The total thickness of the spherical microcavity, including the thickness of the cell-membrane, is roughly $4000 \mathrm{~nm}$; the cell size (diameter) is $8000 \mathrm{~nm}$. 
The spherical microcavity implies "high" resonance around wavelength $300 \mathrm{~nm}$. A resonance in a cavity implies "excessive" transfer of (photon) energy at the computed wavelength. (In the present study, it suffices to qualify the meeting of the condition of the occurrence of resonance in a microcavity as "high" or "good" or "fair".) In reality, a band of wavelengths between $295 \mathrm{~nm}$ to $304 \mathrm{~nm}$ reveal resonance from "good"-to-"high"-to-"good". These wavelengths of resonance are in the UVB band, by definition.

It may be noted that one cannot design an ideal spherical microcavity at $1050 \mathrm{~nm}$ for the virus-core of radius $r=50 \mathrm{~nm}$ (diameter $100 \mathrm{~nm}$ ), since the virus-core size $(100 \mathrm{~nm})$ is not roughly equal to the designed virus-layer thickness $\mathrm{d} \approx 181 \mathrm{~nm}$ at $1050 \mathrm{~nm}$.

(iv) Virus radius $r=100 \mathrm{~nm}$, cell refractive index $n=1.39$

The design of an ideal spherical microcavity at $1050 \mathrm{~nm}$ of viruses of radius $r=100 \mathrm{~nm}$ (diameter $200 \mathrm{~nm}$ ) and refractive index $\mathrm{n}=1.45$ in a cell of refractive index $\mathrm{n}=1.39$ is considered.

The thickness of virus-layer is $\mathrm{d}=1050 / 4 * 1.45 \approx 181 \mathrm{~nm}$, which approximately equals the size of virus-core (diameter) of $200 \mathrm{~nm}$. The thickness of cell-layer is $d=1050 / 4 * 1.39 \approx 189$ $\mathrm{nm}$. There are 10 viruses in a cell. The total thickness of the spherical microcavity, including the thickness of the cell-membrane, is roughly $4000 \mathrm{~nm}$; the cell size (diameter) is $8000 \mathrm{~nm}$.

The spherical microcavity implies "good" resonance around wavelength $350 \mathrm{~nm}$. A resonance in a cavity implies "excessive" transfer of (photon) energy at the computed wavelength. (In the present study, it suffices to qualify the meeting of the condition of the occurrence of resonance in a microcavity as "high" or "good" or "fair".) In reality, a band of wavelengths between $344 \mathrm{~nm}$ to $357 \mathrm{~nm}$ reveal "good" resonance. These wavelengths of resonance are in the UVA band, by definition.

\section{Data Analysis}

In a real-world scenario, the formation of viruses in a cell is a random process. One has to consider propagation of ultraviolet electromagnetic waves in a random dielectric medium. The data on the dielectric medium comprising virus and cell need also to be appropriate to ultraviolet wavelengths. The present study is desired in the prevailing dire coronavirus situation. The aim of the study is to adopt the existing dielectric data in an ideal-world scenario, to compute "excessive" transfer of (photon) energy to the dielectric medium of cells-plus-viruses, if it exists, compared to "normal" delivery of energy by ultraviolet electromagnetic waves to cells alone. Such a study may imply "excessive" delivery of energy by ultraviolet electromagnetic waves to cells-plus-viruses in a real world scenario, which is the need of the hour.

The virus sizes of $100 \mathrm{~nm}$ and 200 are reasonable estimates of small- and large-size virus. The refractive index of 1.45 of virus is also a reasonable value. The cell refractive indices of 1.37 and 1.39 are appropriate to UVB - UVA band of wavelengths $(280-400 \mathrm{~nm})$. (The dielectric medium is not an intrinsically absorbing medium of light. Therefore, refractive index increases with decreasing wavelength of light. In an intrinsically absorbing medium, refractive index 
decreases with decreasing wavelength. Thus, in the present study, the refractive index of cell is also taken as 1.39 in the UVB - UVA band $280-400 \mathrm{~nm}$.)

\section{Conclusion}

The observations on the refractive index of virus and human cell are known above the ultraviolet band of wavelengths $(100-400 \mathrm{~nm})$. The refractive index of cell may be "logically" increased in the ultraviolet band. The model calculations in the present study adopt values of refractive index of cell of 1.37 and 1.39. The virus of size $\sim 100 \mathrm{~nm}$ and $\sim 200 \mathrm{~nm}$ have been used in the model calculations.

The virus in cells may be disintegrated by "excessive" absorption of ultraviolet light at narrow range of wavelengths in the UVB - UVA band of wavelengths $(280-400 \mathrm{~nm})$. Thus, ultraviolet light therapy may provide relief to patients moderately and severely affected by coronavirus, with "lower" levels and "briefer" periods of ultraviolet radiation exposure. However, the minor affected coronavirus patients may require the "normal" levels and periods of ultraviolet radiation exposure.

The ultraviolet radiation in the UVC band $(100-280 \mathrm{~nm})$ may be utilized for the sanitization of material and equipment at frequently visited places, like a shopping mall or an airport. It is worthwhile to note that the quantum of energy $(\mathrm{h} v, \mathrm{~J})$ at the ultraviolet wavelength $100 \mathrm{~nm}$ is three times higher than the quantum of energy $(\mathrm{hv}, \mathrm{J})$ at the ultraviolet wavelength 300 $\mathrm{nm}$, with the usual notation of symbols.

The ultraviolet device, such as, LED/UV lamp may be suitably utilized. (A UV lamp is a traditional technology.) The level (optical power output, in milliwatts/watts), period of radiation exposure and any side effects of radiation exposure of ultraviolet light therapy can only be provided by experts. The ultraviolet radiation is grievously injurious to eyes. One ought to use "UV filtered" glasses while undertaking the ultraviolet light therapy or sanitization work. The present study is dedicated to the author's team mate of the Second Indian Scientific Expedition to Antarctica in 1982-83. Unfortunately, this team mate lost eyesight in both eyes due to the strong direct and ice-reflected solar ultraviolet radiation, while working at the erstwhile station Dakshin Gangotri on the ice shelf.

\section{Acknowledgement}

The author gratefully acknowledges Dr. B. Madhava Reddy, Dr. Mohan K. Raina, Dr. Hirday N. Dutta and Dr. Arun K. Upadhayaya of the National Physical Laboratory for their helpful comments.

\section{References}

[1] https://www.who.int/health-topics/ultraviolet-radiation\#tab=tab_1.

(Google search: Ultraviolet radiation and health-WHO)

[2] https://www.sunlighten.com/blog/difference-light-therapy-near-infrared-therapy/. https://www.ncbi.nlm.nih.gov/pmc/articles/PMC5653719. 
(Google search: near-infrared light therapy)

[3] https://osf.io/preprints/.

(Google search: P K Pasricha, OSF preprint, A Study toward Disintegration of Coronavirus using Near-infrared Light Therapy)

[4] https://abyss.uoregon.edu/js/glossary/virus.html.

(Google search: size and shape of virus. Quoted in: Encyclopedia Britannica)

[5] Virus-NanoFCM, Angew. Chem. Int. Ed., vol. 55, pages 10239-10243, 2016.

(Google search: Virus-NanoFCM. NanoFCM Inc., D6 Thane Road, Nottingham, UK)

[6] Liu et al., "Cell refractive index for cell biology and disease diagnosis: past, present and future", Lab Chip, vol. 16, pages 634-644, 2016.

(Google search: Lab Chip, vol. 16, pages 634-644, 2016. Royal Society of Chemistry, RSC Publishing, UK)

[7] Steelman et al., "Is the nuclear refractive index lower than cytoplasm? Validation of phase measurements and implications for light scattering technologies", J. Biophotonics, vol. 10, pages 1714-1722, 2017.

(Google search: refractive index of cell at $540-720 \mathrm{~nm}$ )

[8] $\mathrm{Xu}$ et al., "High-quality-factor Bragg onion resonators with omnidirectional reflector cladding”, Opt. Lett., vol. 28, pages 2144-2146, 2003.

[9] Kaliteevski et al., "Optical eigenmodes of a multilayered spherical microcavity", J. Mod. Opt., vol. 48, pages 1503-1516, 2001.

[10] Pasricha, P. K., Numerical, Statistical and Computational Methods for the Physical Sciences With C, two volumes, to be published. 